\title{
LHCb Status, First Physics and Discovery Potential
}

\section{Olaf Steinkamp*, on behalf of the LHCb Collaboration.}

Physik-Institut der Universität Zürich, Winterthurerstr. 190, 8057 Zürich, Switzerland

E-mail: olafs@physik.uzh.ch

\begin{abstract}
The LHCb experiment at CERN's Large Hadron Collider (LHC) will perform precision measurements of $\mathrm{CP}$ violating processes and rare decays of $B$ mesons and other hadrons containing $b$ or $c$ quarks. Of particular interest are observables that exhibit high sensitivity to New Physics through possible contributions from new heavy particles in loop diagrams. By searching for New Physics indirectly by studying the effect of virtual particles in internal loops, LHCb will extend the discovery potential to masses far in excess of those accessible in direct searches at the LHC. In this contribution, a brief overview is given of the $\mathrm{LHCb}$ performance and running experience from the first year of LHC operation, some early physics results are presented, and the discovery potential in selected key channels is illustrated. More detailed accounts of the detector performance and of specific physics topics are given in several other LHCb contributions to these proceedings.
\end{abstract}

Kruger 2010: Workshop on Discovery Physics at the LHC

December 5 - 10, 2010

Kruger National Park, Mpumalanga, South Africa

\footnotetext{
* Speaker.
} 


\section{Introduction}

LHCb is the smallest of the four large experiments at the LHC. At the end of 2010, the collaboration counts about 730 physicists from 54 institutions in 15 countries. At present there are no African institutions amongst its members but an Expression of Interest for the participation of physicists in the LHCb experiment has been signed with the University of Antananarivo, Madagascar.

The main goal of the experiment is the indirect search for New Physics through precision measurements of $\mathrm{CP}$ violating phases and rare heavy-quark decays. Of particular interest are processes that are strongly suppressed in the Standard Model, such as flavour-changing neutral current $b \rightarrow s$ transitions. In these loop mediated processes, New Physics can lead to significant deviations from Standard Model predictions through additional amplitudes involving new heavy particles. These indirect searches extend the discovery potential for new particles to a mass range far beyond that accessible in direct searches. Moreover, observing the pattern of deviations from Standard Model predictions will give insights into the underlying dynamics of the New Physics and will permit the parameters of New Physics models to be constrained. The analysis strategies for six selected key measurements have been described in detail in the LHCb "roadmap" document [1]. They comprise measurements of the CKM angle $\gamma$ from $B \rightarrow D K$ tree decays and from penguin-mediated charmless charged two-body $B$ decays, the measurement of the $B_{s}^{0} \bar{B}_{s}^{0}$ mixing phase $\phi_{s}$, the determination of the branching fraction of the very rare decay $B_{s}^{0} \rightarrow \mu^{+} \mu^{-}$, measurements of angular distributions in the rare decay $B^{0} \rightarrow K^{\star} \mu^{+} \mu^{-}$, and radiative $b \rightarrow s \gamma$ decays.

To exploit best the strongly forward peaked $b \bar{b}$ production cross section at the $\mathrm{LHC}$, the $\mathrm{LHCb}$ detector is laid out as a single-arm forward spectrometer. Its acceptance covers polar angles from $15 \mathrm{mrad}$ to $300 \mathrm{mrad}$ in the bending plane of the spectrometer magnet and $250 \mathrm{mrad}$ in the nonbending plane. This corresponds to a pseudo-rapidity coverage of about $1.9<\eta<4$.9. LHCb captures almost $40 \%$ of the $b \bar{b}$ production cross section at the LHC while covering only about $4 \%$ of the solid angle. Additional benefits of measuring at small polar angles are the large Lorentz boost of the produced $b$ hadrons and the possibility to identify and trigger on particles with moderately high transverse momentum $p_{T}$ of a few $\mathrm{GeV}$.

A vertical cross section through the LHCb detector is shown in Fig. 1, a detailed description can be found in [2]. The detector consists of planar stations lined up along the LHC beam axis over a total length of about $20 \mathrm{~m}$ : A silicon micro-strip vertex detector (Vertex Locator) around the $p p$ interaction region is followed by a first $\mathrm{RICH}$ detector, a tracking system comprising a single tracking station (TT) upstream of the $3.7 \mathrm{Tm}$ dipole magnet and three tracking stations (T1-T3) downstream of the magnet, a second RICH detector, electromagnetic and hadronic calorimeters (SPD/PS, ECAL, HCAL) and finally a muon system (M1-M5). Key performance parameters are an excellent vertex resolution resulting in a proper time resolution of about $50 \mathrm{fs}$, a momentum resolution for charged particles of better than $0.5 \%$ up to above $100 \mathrm{GeV}$, good kaon/pion separation from $2 \mathrm{GeV}$ up to $100 \mathrm{GeV}$, and an efficient trigger both for final states with leptons and for purely hadronic final states. The trigger exploits the generic properties of $b$ hadron decays, namely displaced decay vertices and decay products with large $p_{T}$. The first-level trigger (L0) is implemented in hardware and has a maximal output rate of $1 \mathrm{MHz}$. It identifies high- $p_{T}$ track segments in the muon system and high- $E_{T}$ clusters in the calorimeters. The higher-level triggers (HLT) are 


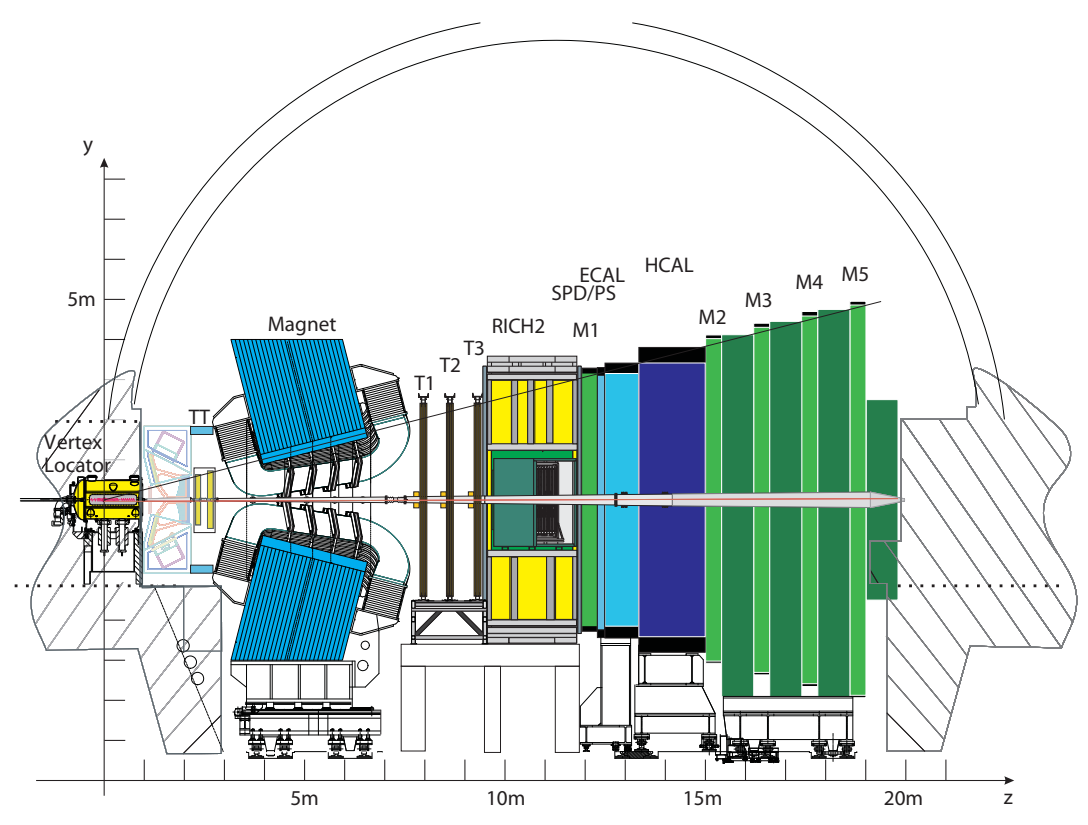

Figure 1: Vertical cross section through the LHCb detector. The $p p$ collision point is in the centre of the Vertex Locator.

implemented in software on a 14,000 core multi-processor computer farm. Initial cuts are made on track impact parameters with respect to the reconstructed primary $p p$ collision vertex and on displaced secondary vertices. Finally, a global event reconstruction is carried out and selections for specific decay channels are performed. Data are saved to disk at a rate of $2 \mathrm{kHz}$.

LHCb was designed to operate at a nominal instantaneous luminosity of $2 \times 10^{32} \mathrm{~cm}^{-2} \mathrm{~s}^{-1}$. For nominal LHC operation, with 2622 colliding proton bunches, this maximizes the fraction of bunch crossings with a single $p p$ interaction ${ }^{1}$. Having a single primary vertex in the event removes the potential risk of assigning the $b$-hadron decay vertex to a wrong primary vertex. Moreover, events with multiple $p p$ interactions tend to suffer from high particle multiplicities and are hard to reconstruct in the very busy forward region covered by LHCb. Its relatively low nominal luminosity makes LHCb a "day-one" experiment at the LHC. For many of its key physics channels, significant measurements can already be expected from the 2010/2011 LHC run.

\section{Detector Performance and Running Experience}

Except for the HLT computing farm, which was only partially installed, the LHCb experiment was fully operational from the first day of LHC collisions. About $37 \mathrm{pb}^{-1}$ of $p p$ collisions at $7 \mathrm{TeV}$ were collected during the $2010 \mathrm{LHC}$ run, with a data taking efficiency of more than $90 \%$. The polarity of the LHCb spectrometer magnet was reversed several times to minimize possible systematics due to detector asymmetries.

\footnotetext{
${ }^{1}$ The nominal instantaneous luminosity for LHCb can be adjusted by an appropriate focusing of the LHC beams in the LHCb collision point, without affecting the other experiments.
} 
LHC running conditions and the instantaneous luminosity provided by the accelerator evolved rapidly throughout the 2010 run. During the earliest runs, data were taken with a minimum-bias trigger. As the LHC luminosity increased, trigger conditions were gradually tightened such that the available readout bandwidth and CPU in the HLT farm were fully exploited. Data were taken at the highest luminosity available from LHC at all times, although during the later part of the run this meant operating the experiment at significantly higher numbers of $p p$ interactions per bunch crossing than foreseen under nominal conditions. Peak instantaneous luminosities close to the LHCb nominal luminosity were achieved towards the end of the 2010 run, but with only 344 instead of 2622 colliding bunches. These running conditions corresponded to an average number of visible interactions per bunch crossing, $\mu$, of up to 2.4 , where nominal LHCb operating conditions correspond to $\mu=0.4$. A visible $p p$ interaction is defined, here, as one that leaves signals in the LHCb detector. It was found that the detector and the reconstruction algorithms were much more robust in these harsh conditions than anticipated. The most significant limitation was found to be CPU time consumption in the not yet fully installed HLT farm for the reconstruction of very busy events. For some trigger lines, cuts on the maximum allowed number of hits in part of the tracking system and in one of the calorimeter sub-systems (SPD) were introduced to veto events with very high particle multiplicities when running at highest $\mu$. The HLT farm has been completed over the 2010/2011 Christmas break. The positive experience from the 2010 creates the interesting option of operating the experiment at a higher than the initially foreseen instantaneous luminosity in 2011.

An excellent vertex resolution is essential for the high-level trigger and for many physics analyses. To minimize extrapolation errors from the first measurement points to the vertex position, the LHCb vertex detector is installed inside the LHC vacuum vessel. The sensitive area of the detector starts at a distance of only $8 \mathrm{~mm}$ from the beam axis during data taking. The detectors have to be retracted by $\pm 3 \mathrm{~cm}$ during beam injection. They are moved into data taking position at the beginning of every fill when stable beams are declared. An internal alignment of better than $5 \mu \mathrm{m}$ of the vertex detector has been obtained. Fill-to-fill variations in the position of the detectors are also as small as $5 \mu \mathrm{m}$. A single-hit resolution of $4 \mu \mathrm{m}$ has been measured for the innermost readout strips. The measured track impact parameter resolution is slightly worse than expected from simulation, possible reasons for this are being investigated.

Excellent momentum and invariant mass resolutions are crucial for the rejection of combinatorial backgrounds. Measured spatial resolutions in the tracking system are approaching those expected from test beams and simulation. Small differences are remaining from residual misalignments. The acceptance of the LHCb tracking system for cosmics being negligibly small, its spatial alignment relies entirely on beam data. The good progress in the understanding of the alignment is demonstrated by the invariant mass resolution obtained for $J / \psi \rightarrow \mu^{+} \mu^{-}$decays, which was 17.1 MeV in May 2010 and had reached $13.3 \mathrm{MeV}$ by the time of the Kruger 2010 workshop. From simulation, an invariant mass resolution of $12.1 \mathrm{MeV}$ is expected. Fig. 2 shows a $\mu^{+} \mu^{-}$ invariant mass distribution in the mass range of the $\Upsilon(1 s), \Upsilon(2 s)$ and $\Upsilon(3 s)$ resonances. An invariant mass resolution of $47 \mathrm{MeV}$ is obtained, here, and the three resonances are clearly resolved. Invariant mass distributions for a few $B \rightarrow J / \psi X$ decay channels are shown in Fig. 3. Applying a mass constraint on the $J / \psi$ mass, resolutions around $10 \mathrm{MeV}$ are obtained for the $B$ mass.

Another crucial ingredient for many analyses is an excellent kaon/pion separation over a wide momentum range. LHCb employs two RICH detectors incorporating three different radiators to 


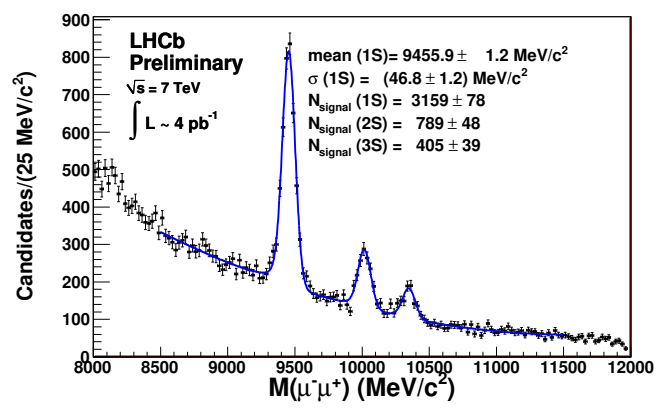

Figure 2: $\mu^{+} \mu^{-}$invariant mass distribution showing the $\Upsilon(1 s), \Upsilon(2 s)$ and $\Upsilon(3 s)$ resonances. 4 pb $^{-1}$ were used to obtain this distribution.
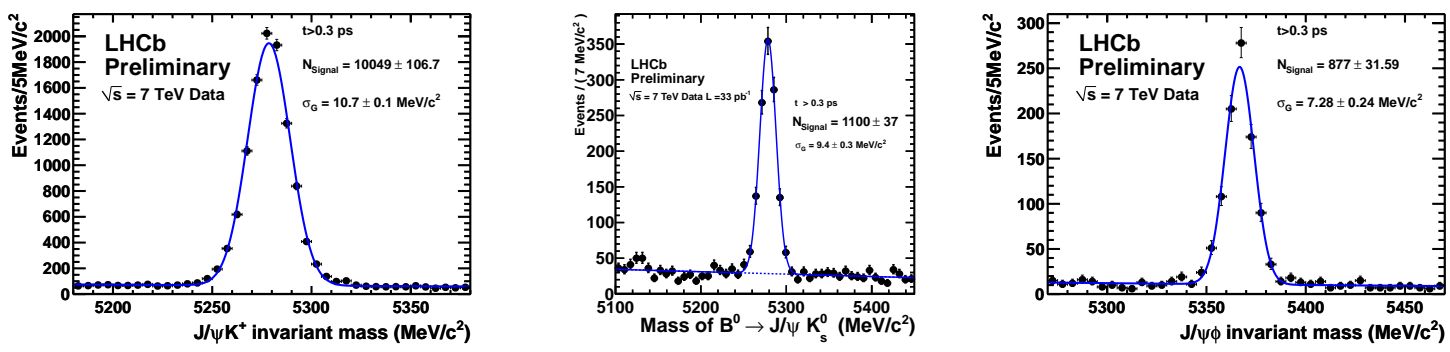

Figure 3: Invariant mass distributions for $B^{+} \rightarrow J / \psi K^{+}$(left), $B^{0} \rightarrow J / \psi K_{s}^{0}$ (middle) and $B_{s}^{0} \rightarrow J / \psi \phi$ (right). A lifetime cut was applied on the $B$ decay vertex to suppress combinatorial background. The $J / \psi$ was reconstructed in the $\mu^{+} \mu^{-}$final state and a mass constraint was applied on the $J / \psi$ mass.

cover momenta down to about $2 \mathrm{GeV}$, needed to identify kaons for $B$ flavour tagging, and up to $100 \mathrm{GeV}$, required for a clean separation of two-body hadronic $B$ decays. The particle-identification performance has been studied on data using tag-and-probe methods on $\phi \rightarrow K^{+} K^{-}, K_{s}^{0} \rightarrow \pi^{+} \pi^{-}$ and $\Lambda \rightarrow p \pi$ decays and is found to be close to expectations from simulation over the full momentum range. The importance of kaon/pion separation for two-body hadronic $B$ decays is illustrated in Fig. 4. The top left plot shows a $B \rightarrow h^{+} h^{\prime-}$ invariant mass spectrum before particle-identification cuts, where the pion mass hypothesis was applied for both final-state particles. Despite the good invariant mass resolution, the contributions from $B^{0} \rightarrow \pi^{+} \pi^{-}, B \rightarrow K^{ \pm} \pi^{\mp}$ and $B_{s}^{0} \rightarrow K^{+} K^{-}$cannot be separated kinematically. The other three plots show invariant mass spectra for each of the three final states after particle-identification cuts have been applied. Clean signals with excellent invariant-mass resolution of $23 \mathrm{MeV}$ are observed for all three decay modes. In the $K^{ \pm} \pi^{\mp}$ final state, contributions from both $B^{0}$ and $B_{s}^{0}$ can be seen.

Finally, the performance of the electromagnetic calorimeter in electron and photon reconstruction is illustrated in Fig. 5, which shows an $e^{+} e^{-}$invariant mass distribution with clear $J / \psi$ and $\psi(2 s)$ signals on the left and a $\chi_{c 1,2} \rightarrow J / \psi \gamma$ signal on the right.

More details on the $\mathrm{LHCb}$ running experience and the performance of the detector, the triggers and the data acquisiton system can be found in another contribution to these proceedings [3].

\section{Early Physics Results}

Due to its unique $\eta$ coverage, its excellent particle identification capabilities and the possibil- 

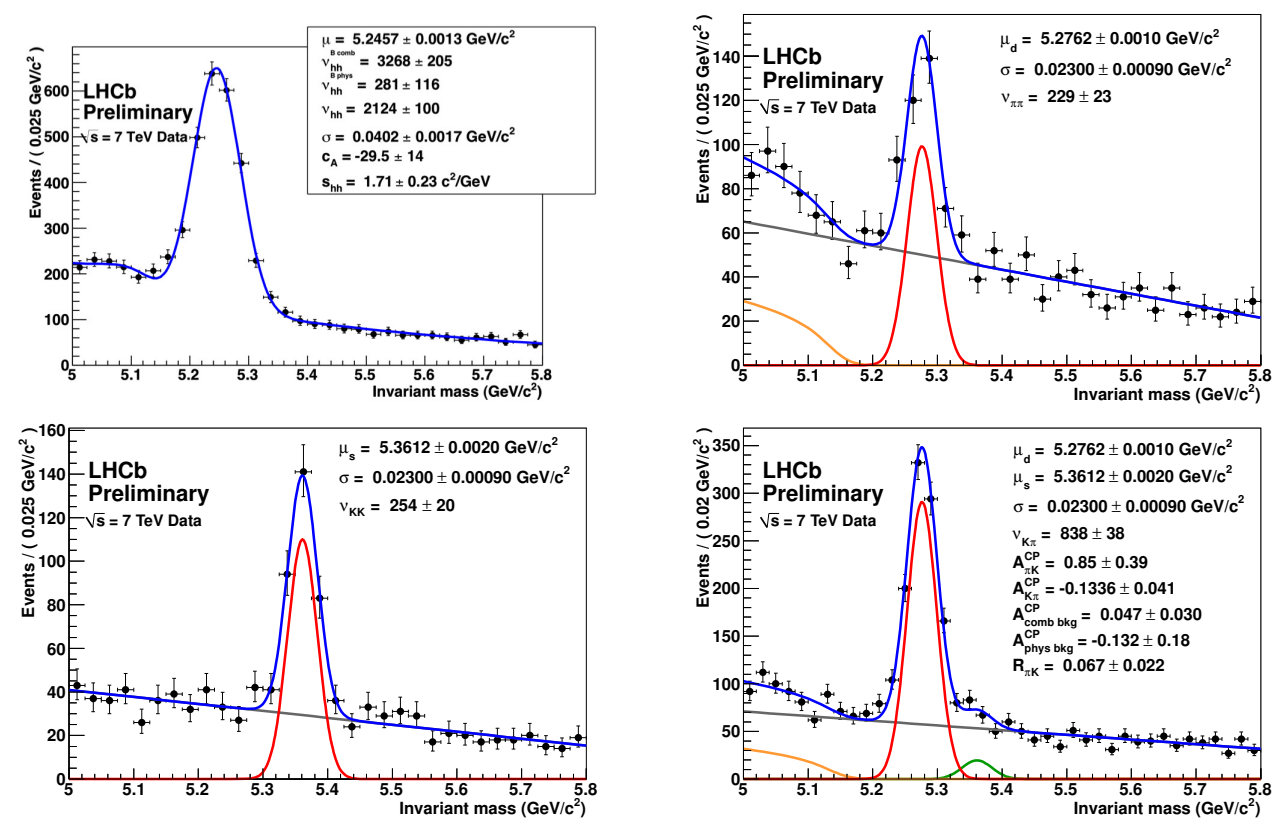

Figure 4: $B \rightarrow h^{+} h^{-}$invariant mass distributions: before particle-identification, where the pion mass hypothesis was applied for both final-state particles (top left), and for $B^{0} \rightarrow \pi^{+} \pi^{-}$(top right), $B_{s}^{0} \rightarrow K^{+} K^{-}$ (bottom left) and $B_{d, s}^{0} \rightarrow K^{ \pm} \pi^{\mp}$ (bottom right) after the application of loose kaon/pion identification criteria. The rising background at low invariant masses is due to mis-identified 3-body decays.
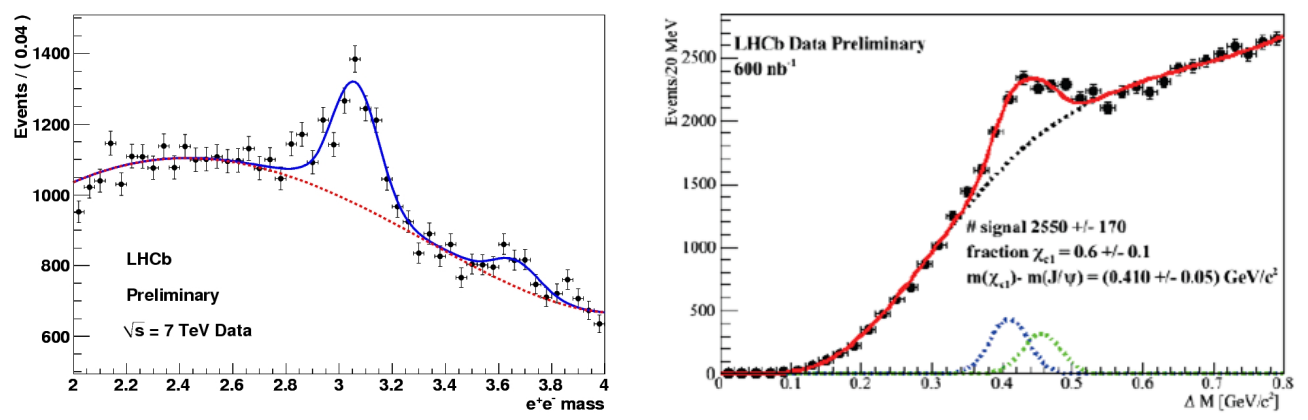

Figure 5: $e^{+} e^{-}$invariant mass distribution showing the $J / \psi$ and $\psi(2 s)$ resonances (left), $\chi_{c 1,2} \rightarrow$ $J / \psi\left(\mu^{+} \mu^{-}\right) \gamma$ signal (right). These distributions were obtained using only $1 \mathrm{pb}^{-1}$ of data or less.

ity to trigger on relatively low- $p_{T}$ particles, LHCb offers interesting opportunities for a wide range of measurements beyond its core physics programme. Studies of strangeness production and of anti-particle/particle production ratios in the forward region are examples of interesting early measurements. They test theoretical models of strangeness production, baryon number transport and hadronisation mechanisms and provide valuable input for the tuning of Monte-Carlo generators. Data were recorded at $\sqrt{s}=0.9 \mathrm{TeV}$ and at $\sqrt{s}=7 \mathrm{TeV}$ during the early days of the 2010 run. A minimum-bias trigger was applied requiring a minimum energy deposit in the calorimeters or at least one track reconstructed in the event. As an example, a comparison of preliminary results on the $\bar{\Lambda} / \Lambda$ production ratio with Monte-Carlo predictions and with an earlier measurement by the STAR collaboration is shown in Fig. 6. The agreement with the earlier measurement is good. 

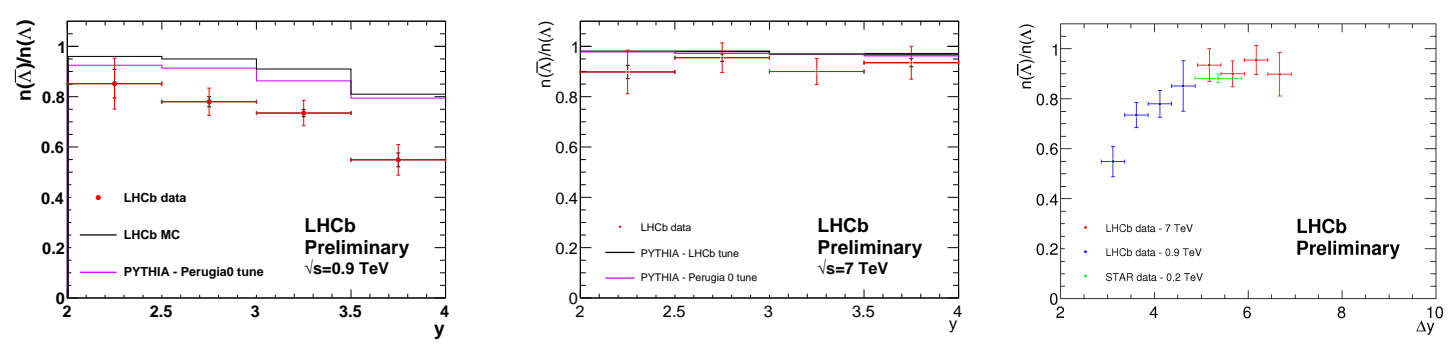

Figure 6: Preliminary results on the $\bar{\Lambda} / \Lambda$ production ratio as a function of the rapidity $y$ at $\sqrt{s}=0.9 \mathrm{TeV}$ (left) and at $\sqrt{s}=7 \mathrm{TeV}$ (middle), compared to predictions from two different tunes of the Pythia MonteCarlo generator; the same $\bar{\Lambda} / \Lambda$ production ratio as a function of the rapidity loss $\Delta y=y_{\text {beam }}-y_{\Lambda}$, compared to a previous measurement by the STAR collaboration (right).

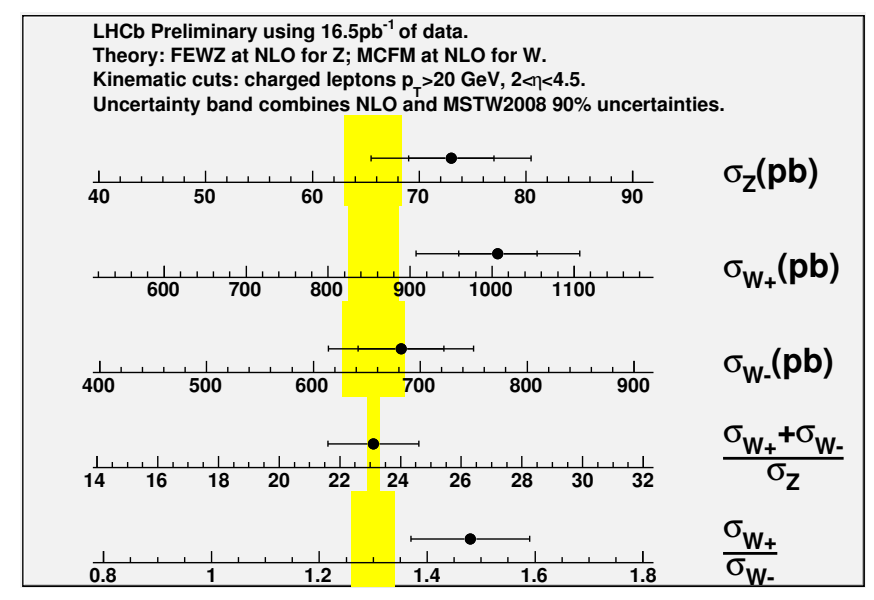

Figure 7: Summary of $W$ and $Z$ production cross sections and cross section ratios in the pseudo-rapidity range $2<\eta$ (charged leptons) $<4$.5. Points and error bars are preliminary LHCb results from $16.5 \mathrm{pb}^{-1}$ of data collected at $\sqrt{s}=7 \mathrm{TeV}$, the vertical band indicates theory predictions from NLO calculations.

The Monte-Carlo describes well the observed distribution at $\sqrt{s}=7 \mathrm{TeV}$ but clearly overestimates the ratio at $\sqrt{s}=0.9 \mathrm{TeV}$. Further results from these studies are summarized in another LHCb contribution to these proceedings [4].

In the field of electro-weak physics, LHCb offers the opportunity to study $W, Z$ and DrellYan production in the interesting forward region and to determine parton density functions of the proton in a previously unexplored kinematical region. Preliminary results from the analysis of $16.5 \mathrm{pb}^{-1}$ of data on $Z^{0}, W^{+}$and $W^{-}$production cross sections, the $W / Z$ production ratio and the $W^{+} / W^{-}$production asymmetry were for the first time presented at Kruger 2010 . These results are summarized in Fig. 7. They are in good agreement with predictions from theory and provide new constraints on proton parton density functions at low momentum fraction $x$ and high momentum transfer $q^{2}$. More details on these analyses are given in another LHCb contribution to these proceedings [5]. Studies of Drell-Yan production to extend these measurements to low values of $q^{2}$ are also ongoing.

Valuable information on the gluon density $x G\left(x, q^{2}\right)$ in the regime of low momentum fraction $x$ can be obtained from measurements of forward jet production at LHCb. Preliminary studies of 

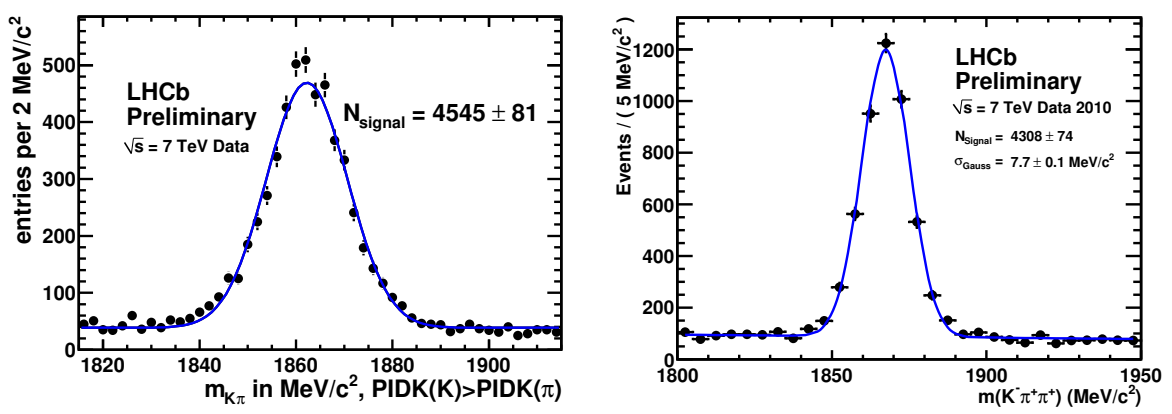

Figure 8: $K^{+} \pi^{-}$invariant mass distribution showing a clear $D^{0}$ signal (left) and $K^{-} \pi^{+} \pi^{+}$invariant mass distribution showing a clear $D^{+}$signal (right). These distributions were obtained using only $1.8 \mathrm{nb}^{-1}$ of data.

inclusive jet distributions and dijet characteristics are underway. Interesting results on perturbative QCD at $x \approx 10^{-4}$ are expected from the 2010 data. These analyses are described in more detail in another LHCb contribution to these proceedings [6].

The same features that make LHCb an excellent $b$-physics experiment make it very well suited for charm physics. The charm production cross section being about 20 times larger at the LHC than the $b$ cross section, large event samples can be collected. The study of secondary charm production from $b$ decays is of significant interest for the $\mathrm{LHCb} b$ physics programme.

Preliminary measurements of differential cross sections for prompt open charm production to $D^{0}, D^{ \pm}, D^{\star \pm}$ and $D_{s}^{ \pm}$have been performed using $1.8 \mathrm{nb}^{-1}$ of data. The $D$ mesons were reconstructed in hadronic final states. As illustrated in Fig. 8 for the example of $D^{0} \rightarrow K^{+} \pi^{-}$and $D^{+} \rightarrow K^{-} \pi^{+} \pi^{+}$, clean event samples are obtained thanks to the excellent particle identification. A cut on the $D$ impact parameter with respect to the primary vertex is employed to separate the prompt component from secondary $D$ mesons from $b$ decays. Differential cross sections for prompt production are measured in two-dimensional bins of pseudo-rapidity in the range $2<\eta<4.5$ and transverse momentum from $0<p_{T}<8 \mathrm{GeV}$. Results are in good agreement with predictions from theory. More details on these analyses and the preliminary results are given in Ref. [7] and in another LHCb contribution to these proceedings [8]. The analysis of a larger event sample is underway.

Measurements of differential cross sections for prompt $J / \psi$ and $b \rightarrow J / \psi X$ production have been performed using $5.2 \mathrm{pb}^{-1}$ of data. Preliminary results from this analysis were presented for the first time at Kruger 2010 and are summarized in another contribution to these proceedings [9]. Since the workshop, these results have been submitted as a journal publication [10]. The measurement was performed in two-dimensional bins of pseudo-rapidity from $2<\eta<4.5$ and transverse momentum from $0<p_{T}<14 \mathrm{GeV}$. To separate prompt $J / \psi$ and $J / \psi$ from $b$ decays, the pseudo proper time $t_{z}=\Delta z \cdot M_{J / \psi} / p_{z}$ was used, where $\Delta z$ is the displacement of the $J / \psi$ vertex from the primary vertex along the beam axis, $M_{J / \psi}$ is the $J / \psi$ mass and $p_{z}$ is the component of the $J / \psi$ momentum along the beam axis. The distribution of $t_{z}$ in one of the $\left(p_{T}, \eta\right)$ bins used in the analysis is shown in Fig. 9. Combinatorial backgrounds in the $t_{Z}$ distribution were estimated by combining $J / \psi$ vertices from one event with primary vertices from a different event. The dominating uncertainty on the prompt $J / \psi$ cross section is due to the still unknown $J / \psi$ polar- 

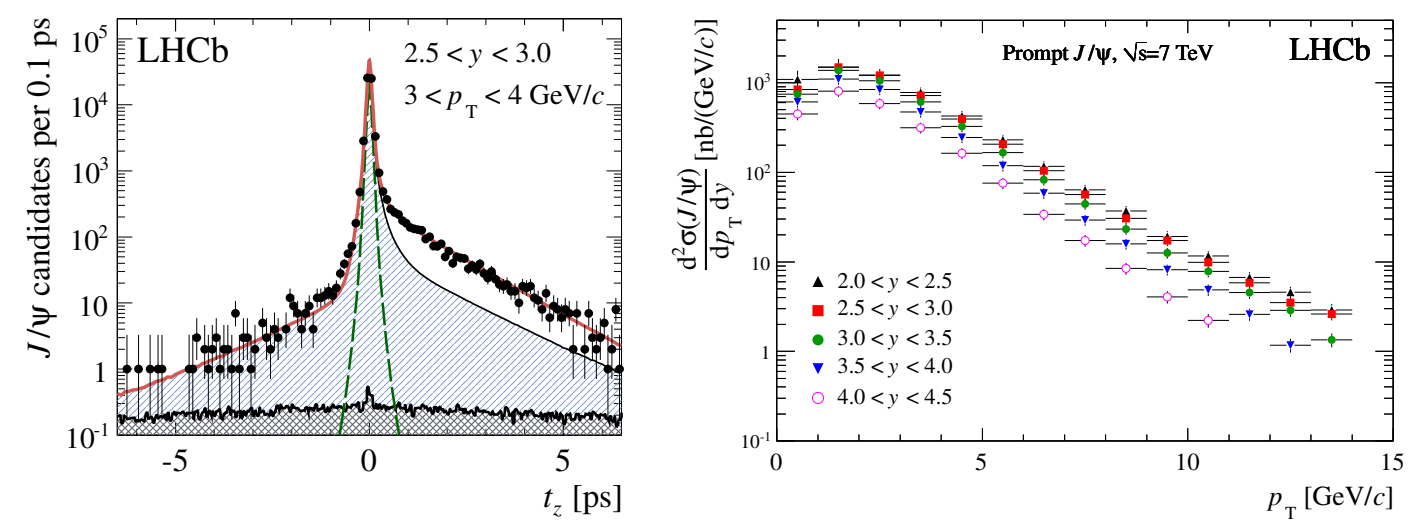

Figure 9: Distribution of the $J / \psi$ "pseudo proper time" $t_{z}$ in one of the $\left(p_{T}, \eta\right)$ bins used in the measurement of differential $J / \psi$ cross sections. See the text for a definition of $t_{z}$ (left). Prompt $J / \psi$ production crosssection as a function of $p_{T}$ in bins of $y$, assuming no polarization of the prompt $J / \psi$ (right). These results are based on $5.2 \mathrm{pb}^{-1}$ of data.

ization in prompt production. The measurement of the $J / \psi$ polarization by $\mathrm{LHCb}$ will require a larger data sample. The differential cross section assuming unpolarized $J / \psi$ production is shown in Fig. 9. The results are in good general agreement with recent theoretical calculations at high $p_{T}$, although the uncertainties on these predictions are still rather large. The LHCb Monte Carlo simulation, based on PYTHIA 6.4 [11] and EvtGen [12], was used to extrapolate the measured $b \rightarrow J / \psi X$ cross section from the LHCb acceptance to the full polar angle range. Using the average $b \rightarrow J / \psi X$ branching fraction for inclusive $b$-hadron decays to $J / \psi$ measured at LEP [13], a $b \bar{b}$ production cross section of $\sigma(p p \rightarrow b \bar{b} X)=288 \pm 4 \pm 48 \mu \mathrm{b}$ is calculated. This result is in excellent agreement with an earlier LHCb measurement of the $b \bar{b}$ production cross section that was based on $15 \mathrm{nb}^{-1}$ of data and used $B^{0} \rightarrow D^{0} \mu^{-} X^{+}$decays [14]. In this analysis, the impact parameter of the reconstructed $D^{0}$ momentum with respect to the primary vertex was used to identify $D$ mesons from $b$ decays and wrong-sign $D^{0} \mu^{+}$combinations were used to estimate backgrounds. Using the LHCb Monte Carlo simulation to extrapolate from the LHCb acceptance to the full polar angle range and using fragmentation fractions measured at LEP, a $b \bar{b}$ production cross section of $\sigma(p p \rightarrow b \bar{b} X)=284 \pm 20 \pm 49 \mu \mathrm{b}$ was calculated, in good agreement with the newer and more precise measurement. Prior to these measurements, simulation studies assessing the LHCb physics reach had assumed a $b \bar{b}$ production cross section of $250 \mu \mathrm{b}$ at $\sqrt{s}=7 \mathrm{TeV}$.

A measurement of direct $\mathrm{CP}$ violation in charged charmless hadronic two-body decays is underway. Competitive results with previous measurements from the $B$ factories and the Tevatron can be expected from the 2010 data sample. An invariant mass distribution for $B_{d, s}^{0} \rightarrow K^{ \pm} \pi^{\mp}$ decays was already shown in Fig. 4 above. Separating the event sample further into $B_{d, s}^{0} \rightarrow K^{+} \pi^{-}$and $\bar{B}_{d, s}^{0} \rightarrow K^{-} \pi^{+}$, a clear difference in event yields is visible in the invariant mass spectra shown in Fig. 10. The raw asymmetry between the $B^{0}$ and $\bar{B}^{0}$ yields deviates from zero at the level of three standard deviations and is compatible with the known world average CP asymmetry. An asymmetry is also visible in the $B_{s}^{0}$ vs. $\bar{B}_{s}^{0}$ event yields although the effect is not yet statistically significant, here. The distributions shown here are not corrected for production and detection asymmetries. These corrections are, however, expected to be small. 

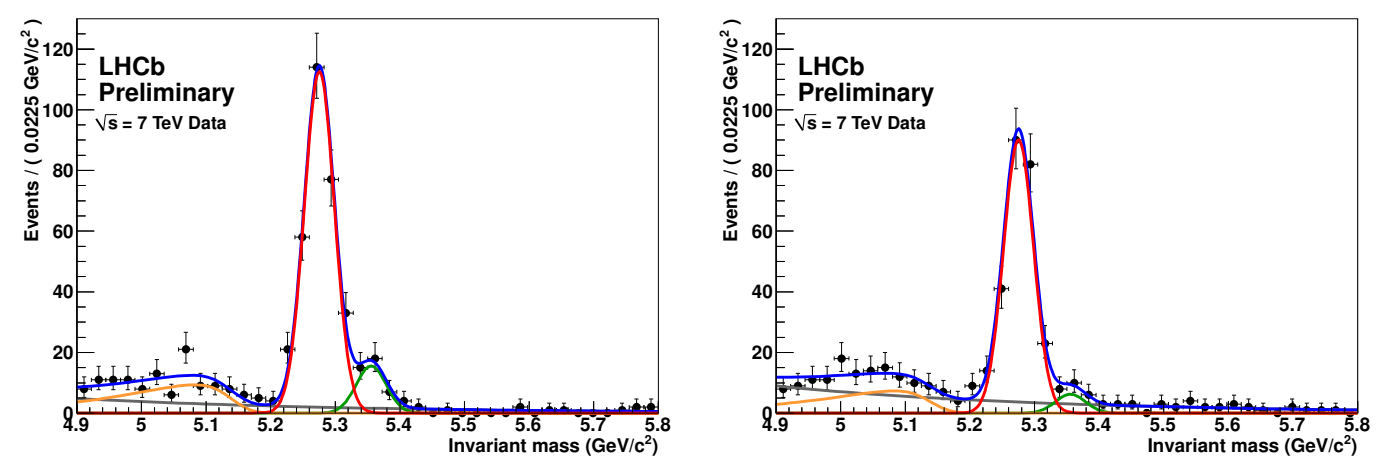

Figure 10: Invariant mass distributions for $B_{d}^{0} / B_{s}^{0} \rightarrow K^{+} \pi^{-}$(left) and $\bar{B}_{d}^{0} / \bar{B}_{s}^{0} \rightarrow K^{-} \pi^{+}$(right). The red line indicates the fitted signal from $B_{d}^{0}$ resp. $\bar{B}_{d}^{0}$ decays, the green line indicates the contribution from $B_{s}^{0}$ resp. $\bar{B}_{s}^{0}$ decays. The yellow and green lines indicate backgrounds from mis-identified three-body decays and combinatorial backgrounds, respectively. The shown distributions have not been corrected for production asymmetries and detector asymmetries.

The ability to resolve the fast $B_{s}^{0} \bar{B}_{s}^{0}$ flavour oscillations is an important ingredient for several LHCb key measurements. A measurement of the $B_{s}^{0} \bar{B}_{s}^{0}$ oscillation frequency in $B_{s}^{0} \rightarrow D_{s}^{-}(3) \pi$ decay modes from the 2010 data is being prepared for the 2011 winter conferences. Despite the still limited statistics, a competitive measurement with existing results from the Tevatron can be expected due to the excellent proper-time resolution of the experiment. Another important ingredient for oscillation measurements is the ability to tag the initial flavour of the $B$ meson at production. Opposite-side lepton, kaon and vertex-charge tags make use of distinctive signatures from the decay of the accompanying $b$ hadron in the event to imply the flavour of the $B$ meson under study. Sameside tags directly determine the flavour of the $B$ meson under study, using the charge of a pion, in the $B^{0}$ case, or a kaon, in that of the $B_{s}^{0}$, from the $b$ quark fragmentation chain or from the decay of excited $B$ states. Flavour-specific decay channels such as $B^{+} \rightarrow J / \psi K^{+}, B^{0} \rightarrow J / \psi K^{\star}\left(K^{+} \pi^{-}\right)$ and $B^{0} \rightarrow D^{0} \mu^{+} v_{\mu}$ are employed to optimize and calibrate the flavour-tagging algorithms. This calibration is still ongoing. As an example of the tagging performance at the time of Kruger 2010, Fig. 11 shows the observed flavour asymmetry as a function of the $B$ meson proper time for decays $B^{0} \rightarrow D^{0} \mu^{+} v_{\mu}$. The initial flavour at production was determined using opposite side lepton and kaon tags, the flavour at decay is given by the charge of the final state muon. The flavour oscillation is clearly visible and in good agreement with the known $B^{0} \bar{B}^{0}$ oscillation frequency $\Delta m_{d}$.

\section{Physics Goals and Potential: CP Violation}

The CKM angle $\gamma=\arg \left(\left(V_{u d} V_{u b}^{\star}\right) /\left(V_{c d} V_{c b}^{\star}\right)\right)$ is the most difficult to measure angle of the unitarity triangle and therefore the one least well constrained by direct observations. A precise measurement of this angle requires several key features of the LHCb experiment, such as large $B$ meson yields, efficient triggers for purely hadronic final states and excellent kaon/pion identification.

A theoretically clean determination of $\gamma$ can be obtained through the measurement of time integrated decay rates for tree decays $B^{ \pm} \rightarrow D^{0}\left(\bar{D}^{0}\right) K^{ \pm} \rightarrow f K^{ \pm}$, where $f$ is a common final state accessible to both $D^{0}$ and $\bar{D}^{0}$. Sensitivity to $\gamma$ arises through the interference of the tree amplitudes 


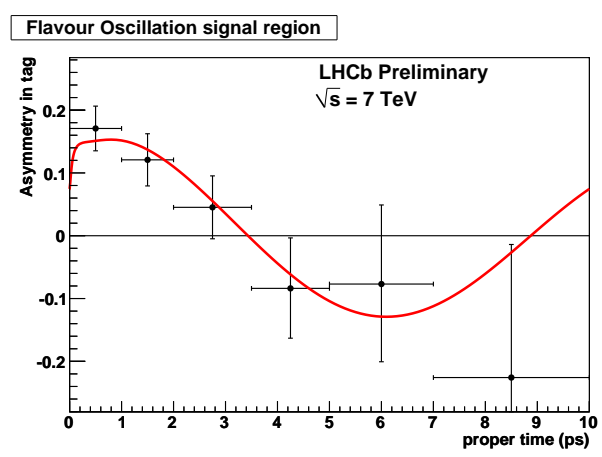

Figure 11: Flavour asymmetry as a function of the $B$ proper time in $B \rightarrow D \mu v_{\mu}$ decays. Opposite-side lepton and kaon taggers were used to tag the initial flavour of the $B$ meson. The overlayed fit function was obtained fixing $\Delta m_{d}$ to its PDG value. $1.9 \mathrm{pb}^{-1}$ were used to obtain this distribution.

Table 1: Expected event yields for $B^{ \pm} \rightarrow D K^{ \pm}$decays from $1 \mathrm{fb}^{-1}$ of integrated luminosity at $\sqrt{s}=7 \mathrm{TeV}$.

\begin{tabular}{|c|c|c|}
\hline method & decay modes & expected yields \\
\hline GLW & $B^{ \pm} \rightarrow D\left(K^{ \pm} K^{\mp}\right) K^{ \pm} ; B^{ \pm} \rightarrow D\left(\pi^{ \pm} \pi^{\mp}\right) K^{ \pm}$ & $2000 ; 750$ \\
\hline ADS & $B^{ \pm} \rightarrow D\left(K^{\mp} \pi^{ \pm}\right) K^{ \pm} ; B^{ \pm} \rightarrow D\left(K^{ \pm} \pi^{\mp}\right) K^{ \pm}$ & $400 ; 20000$ \\
\hline GGSZ & $B^{ \pm} \rightarrow D\left(K_{s}^{0} \pi^{ \pm} \pi^{\mp}\right) K^{ \pm}$ & $>1600$ \\
\hline
\end{tabular}

with a $D^{0}$ and a $\bar{D}^{0}$ in the intermediate state. Different final states $f$ have been proposed: CP eigenstates $K^{ \pm} K^{\mp}$ and $\pi^{ \pm} \pi^{\mp}$ (GLW [15]), Cabibbo favoured / doubly Cabibbo suppressed states $K^{ \pm} \pi^{\mp}$ (ADS [16]), and Dalitz decays $K_{s}^{0} \pi^{ \pm} \pi^{\mp}$ (GGSZ [17]). The GLW method requires to measure two decay rates, the ADS method to measure four decay rates, of which two are Cabibbo favoured and two doubly Cabibbo suppressed. The GGSZ requires the analysis of differences in the Dalitz plots of $D$ meson decays from $B^{+} \rightarrow D K^{+}$and from $B^{-} \rightarrow D K^{-}$. LHCb is going to perform measurements in all these modes. Clean signals are observed in the 2010 data and the measured rates match expectations from simulation. The expected event yields for $1 \mathrm{fb}^{-1}$ of data at $\sqrt{s}=7 \mathrm{TeV}$ are summarized in Tab. 1. Statistics for the GLW and ADS analyses can be further increased by adding self-tagging $B^{0}$ decay modes $B^{0} \rightarrow D^{0}\left(\bar{D}^{0}\right) K^{\star} \rightarrow f K^{\star}$ and by adding other $D$ decay modes, such as $D \rightarrow K 3 \pi$ for the ADS case. A clean determination of $\gamma$ can also be obtained through measurements of time-dependent $\mathrm{CP}$ asymmetries in the tree decays $B^{0} \rightarrow D^{ \pm} \pi^{\mp}$ and $B_{s}^{0} \rightarrow D_{s}^{ \pm} K^{\mp}$. Here, sensitivity to weak phases arises through the interference of $B^{0}\left(\bar{B}^{0}\right)$ resp. $B_{s}^{0}\left(\bar{B}_{s}^{0}\right)$ decays to the same final state with and without mixing. The measured phases are $\gamma+2 \beta$ and $\gamma-\phi_{s}$, respectively. The CKM angle $\gamma$ can then be determined using precise measurements of $2 \beta$ from $B^{0} \rightarrow J / \psi K_{s}^{0}$ and $\phi_{s}$ from $B_{s}^{0} \rightarrow J / \psi \phi$. Combining all time-integrated and time-dependent measurements, a determination of $\gamma$ from tree decays with a precision of about 7-9 can be expected from $1 \mathrm{fb}^{-1}$ of integrated luminosity.

Within the Standard Model, and assuming symmetry of the strong interaction under the exchange of $d$ and $s$ quarks ( $U$-spin symmetry), a clean determination of $\gamma$ can also be obtained from a combined measurement of time-dependent CP asymmetries in $B^{0} \rightarrow \pi^{+} \pi^{-}$and $B_{s}^{0} \rightarrow K^{+} K^{-}$. These decays are, however, penguin-mediated and can therefore be affected by possible contri- 
butions from New Physics in the penguin loops. Due to these New Physics contributions, the value of " $\gamma$ " extracted from this measurement could differ appreciably from the one determined in $B \rightarrow D K$ tree decays. A comparison of the results of the two analyses therefore provides an interesting probe for New Physics. As already illustrated in Fig. 4 above, clean signals for both $B^{0} \rightarrow \pi^{+} \pi^{-}$and $B_{s}^{0} \rightarrow K^{+} K^{-}$are obtained. The measured event yields match expectations from simulation. A measurement of " $\gamma$ " from penguins with a precision of about $15^{\circ}$ can be expected from an integrated luminosity of $1 \mathrm{fb}^{-1}$ at $\sqrt{s}=7 \mathrm{TeV}$. The prospects for $\gamma$ measurements from tree decays and from penguin decays are described in more detail in another LHCb contribution to these proceedings [18].

The precise determination of the CP violating phase $\phi_{s}$ in $B_{s}^{0} \rightarrow J / \psi \phi$ is another key measurement for LHCb. CP violation in this decay is generated through the interference of $B_{s}^{0}\left(\bar{B}_{s}^{0}\right)$ decays to the same final state either directly or after mixing. The phase is predicted to be small in the Standard Model, $\phi_{s}=-2 \arg \left(\left(\left(V_{t s} V_{t b}^{\star}\right) /\left(V_{c s} V_{c b}^{\star}\right)\right)=-(0.0363 \pm 0.0017)\right.$ rad, but can be significantly larger if New Physics contribute to the box diagram mediating $B_{s}^{0} \bar{B}_{s}^{0}$ mixing. Experimentally, $\phi_{s}$ is best determined from the measurement of the time-dependent $\mathrm{CP}$ asymmetry in $B_{s}^{0} \rightarrow J / \psi \phi$. The analysis is complicated by the fact that $B_{s}^{0} \rightarrow J / \psi \phi$ is a decay of a pseudo-scalar meson to two vector mesons. The relative angular momentum between the two vector mesons can be $\ell=0,1,2$ and the final state $J / \psi \phi$ is thus not a pure $\mathrm{CP}$ eigenstate. A time-dependent angular analysis is required to separate statistically the three contributions. Furthermore, the lifetime difference $\Delta \Gamma_{s}$ between the two CP eigenstates in the $B_{s}^{0} / \bar{B}_{s}^{0}$ system is not precisely known and has to be determined simultaneously with $\phi_{s}$. These complications increase demands on the event statistics that are required for a measurement of $\phi_{s}$. Preliminary results have been reported by CDF [19] and D0 [20], using 6500 events from $5.2 \mathrm{fb}^{-1}$ and 3400 events from $6.1 \mathrm{fb}^{-1}$, respectively. Both results are compatible with the Standard Model expectation at slightly more than one standard deviation in the $\left(\phi_{s}, \Delta \Gamma_{s}\right)$ plane, but uncertainties on these measurements are still large. As illustrated in Fig. 3 above, LHCb observes a clean $B_{s}^{0} \rightarrow J / \psi \phi$ signal in the 2010 data. The event yield is compatible with expectations from simulation and about 30,000 selected events are expected for $1 \mathrm{fb}^{-1}$. Another LHCb key feature for this analysis is the excellent proper-time resolution of 50 fs that permits the fast $B_{s}^{0} \bar{B}_{s}^{0}$ oscillations to be cleanly resolved. Possible biases on the angular distributions due to detector acceptance, trigger and event selection cuts will be corrected from simulation and validated using control channels with a similar decay topology as the signal channel, such as $B^{0} \rightarrow J / \psi K^{\star}$. Several auxiliary analyses, such as a measurement of the $B_{s}^{0} \bar{B}_{s}^{0}$ oscillation frequency $\Delta m_{s}$, are being prepared for the 2011 winter conferences. A world-best measurement of $\phi_{S}$ can be expected from LHCb in 2011. This analysis is also described in another contribution to these proceedings [21].

The power of LHCb as a charm experiment has been briefly illustrated in Sec. 3 above. CP violation in the charm sector is very small in the Standard Model and any observation of a sizeable CP asymmetry would be a clear sign for New Physics. Experimentally, this field is largely unexplored. The most promising observables for early $\mathrm{CP}$ measurements at $\mathrm{LHCb}$ are lifetime asymmetries, for example in $D^{0} \rightarrow K^{+} K^{-}$and $\bar{D}^{0} \rightarrow K^{+} K^{-}$. The flavour of the $D$ meson is tagged by the charge of the slow pion requiring the $D$ to be produced through $D^{\star} \rightarrow D^{0} \pi^{+}$. First competitive lifetime asymmetry measurements can be expected from the 2010 data. Many other analyses are underway and results from direct CP violation searches can be expected for 2011 data. The prospects of LHCb in the charm sector are described in more detail in another contribution to these proceedings [8]. 


\section{Physics Goals and Potential: Rare $B$ Decays}

Flavour-changing neutral current $B$ decays offer a number of promising opportunities for New Physics searches at LHCb. These decays are forbidden at tree level in the Standard Model and can proceed only through loop diagrams. Branching fractions and other observables can be significantly modified with respect to Standard Model predictions due to contributions from New Physics in these loops. LHCb has excellent potential in this field due its good invariant mass resolution and the large $B$ meson samples it will collect.

One of the key measurements for LHCb is the search for the very rare decay $B_{s}^{0} \rightarrow \mu^{+} \mu^{-}$. The branching fraction for this decay is predicted to be as small as $(3.35 \pm 0.32) \times 10^{-9}$ in the Standard Model [22] but can be significantly enhanced in the presence of New Physics. For example, in Minimal Supersymmetric Standard Models, the branching fraction increases with the sixth power of the ratio of the Higgs vacuum expectation values, $\tan \beta$. The decay has not yet been observed. The best upper limits on the branching fraction, reported by the Tevatron experiments [25, 26], are still an order of magnitude above the Standard Model prediction. A measurement of a significantly larger cross section than predicted by the Standard Model would be a clear sign for New Physics. On the other hand, improving the current upper limits on the cross section will constrain the parameter space for New Physics models (for examples, see [23]). An important feature of the LHCb analysis strategy is the extensive use of control channels in order to reduce dependency on simulation. Here, the analysis profits from the large samples of hadronic two-body $B$ decays that $\mathrm{LHCb}$ collects. To avoid the need for luminosity measurements and to reduce systematics from trigger and reconstruction efficiencies, the $B_{s}^{0} \rightarrow \mu^{+} \mu^{-}$branching fraction will be determined relative to a normalization channel with a well known branching fraction. One of the selected normalization channels is $B^{+} \rightarrow J / \psi\left(\mu^{+} \mu^{-}\right) K^{+}$. Its branching fraction has been precisely measured [24] and it is selected by the same trigger as the signal channel. The effect of the additional kaon on the reconstruction efficiency can be determined from data by comparing the reconstruction efficiencies for $B^{+} \rightarrow J / \psi\left(\mu^{+} \mu^{-}\right) K^{+}$and $B^{0} \rightarrow J / \psi\left(\mu^{+} \mu^{-}\right) K^{\star}\left(K^{+} \pi^{-}\right)$, which also differ by one hadron in the final state. The dominating systematic using this normalisation channel is due to the uncertainty on the ratio $f_{s} /\left(f_{u}+f_{d}\right)$ of $b$-quark hadronization fractions into $B_{s}^{0}, B^{+}$and $B^{0}$ mesons. A method for determining $f_{s} / f_{d}$ in a model independent way using the relative yields of $B_{d, s}^{0} \rightarrow D_{d, s}^{ \pm} \pi^{\mp}$ and $B_{d, s}^{0} \rightarrow D_{d, s}^{ \pm} K^{\mp}$ measured by LHCb has been proposed [27]. A precision of 6-9\% on $f_{s} / f_{d}$ from this analysis is expected by summer 2011. Two other normalisation channels with different systematics are $B^{0} \rightarrow K^{+} \pi^{-}$and $B_{s}^{0} \rightarrow J \psi\left(\mu^{+} \mu^{-}\right) \phi\left(K^{+} K^{-}\right)$. The former is selected by a different trigger but is a two-body decay with similar final state topology as the signal channel. The latter has a less well measured branching fraction but does not require knowledge of $f_{s} / f_{d}$. An analysis of the 2010 data is being prepared for the 2011 winter conferences. Indeed, while completing these proceedings, final results from the 2010 data have been announced [28]. An upper limit on the branching fraction close to the best limits reported by the Tevatron experiments is obtained already from this relatively small data set. For $1 \mathrm{fb}^{-1}$, a $90 \%$ exclusion limit approaching the Standard Model branching fraction is expected. A $5 \sigma$ observation of the Standard Model branching fraction will require 6-10 $\mathrm{fb}^{-1}$.

Angular distributions in the rare decay $B^{0} \rightarrow K^{\star} \mu^{+} \mu^{-}$give rise to several observables that are sensitive to contributions from New Physics [29]. The most promising of these is the forward- 

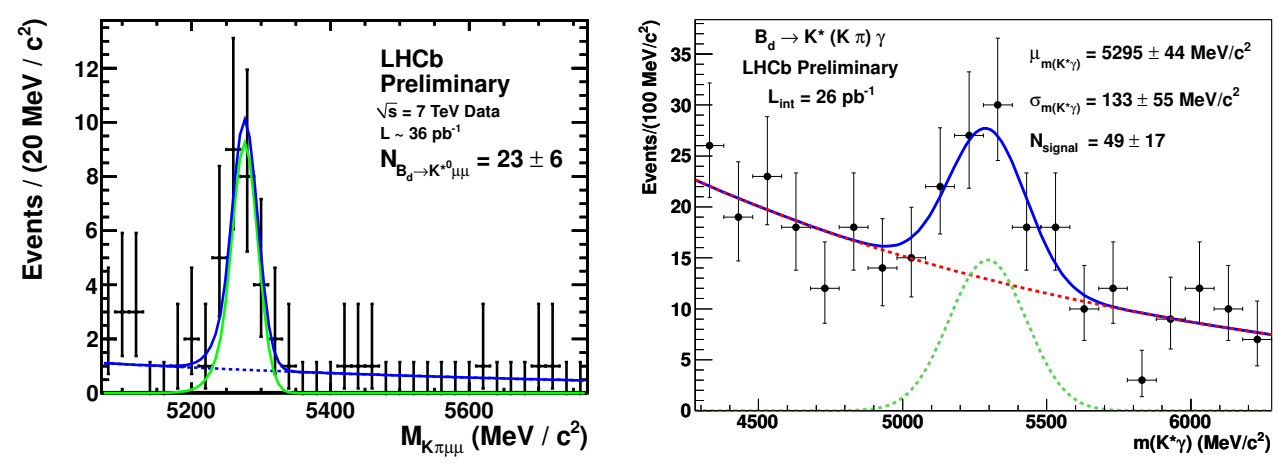

Figure 12: $K \pi \mu \mu$ invariant mass distribution showing a clear $B^{0} \rightarrow K^{\star} \mu^{+} \mu^{-}$signal (left). $K^{\star} \gamma$ invariant mass distribution obtained using $26 \mathrm{pb}^{-1}$ of data (right).

backward asymmetry $A_{F B}$, defined by the angle between the $\mu^{-}$direction and the direction of the $B^{0}$ measured in the rest frame of the $\mu^{+} \mu^{-}$system. In particular the zero-crossing point of $A_{F B}$ as a function of the $\mu^{+} \mu^{-}$invariant mass, $q^{2}$, is precisely predicted in the Standard Model and any observed deviation from this prediction would be a clear sign for New Physics. First measurements of $A_{F B}$ as a function of $q^{2}$ have been published by the BaBar [30], Belle [31] and CDF [32] collaborations. All three measurements find $A_{F B}$ larger than predicted by the Standard Model but the uncertainties on these measurements are still large. As illustrated in Fig. 12, LHCb observes a clear $B^{0} \rightarrow K^{\star} \mu^{+} \mu^{-}$signal in the 2010 data. About 1400 reconstructed events are expected for $1 \mathrm{fb}^{-1}$. One of the most important aspects of the analysis is to correct for possible biases on the angular distributions due to detector acceptance and selection cuts on the momenta and transverse momenta of the daughter particles. These biases will be studied in simulation and validated on data using the large sample of $B^{0} \rightarrow J / \psi\left(\mu^{+} \mu^{-}\right) K^{\star}$ decays that LHCb is going to collect. With only $0.1 \mathrm{fb}^{-1}$ of data, $\mathrm{LHCb}$ will be able to reach a sensitivity on $A_{F B}$ that is similar to the current measurements from the $B$ factories and CDF.

Radiative $b \rightarrow s \gamma$ penguin decays provide a variety of opportunities for New Physics searches. Of particular interest is the measurement of the photon polarization. The Standard Model predicts almost $100 \%$ left-handed polarization, the right-handed component being suppressed by $m_{s} / m_{b}$. Experimentally, the photon polarization can be extracted from measurements of the time dependent decay rate for $B_{s}^{0} \rightarrow \phi \gamma$ and other decays $B_{d, s}^{0} \rightarrow \Phi^{C P} \gamma$ where $\Phi^{C P}$ is a CP eigenstate. Another possibility is to probe the polarization of virtual photons through an angular analysis of $B^{0} \rightarrow$ $K^{\star} e^{+} e^{-}$decays. Another approach to look for New Physics is through the CP asymmetry in $B^{0} \rightarrow$ $K^{\star} \gamma$. A first $B^{0} \rightarrow K^{\star} \gamma$ signal is shown in Fig. 12. Sensitive measurements from radiative decays can be expected from $2 \mathrm{fb}^{-1}$.

A more detailed summary of prospects for New Physics searches in rare $B$ decays at $\mathrm{LHCb}$ can be found in another contribution to these proceedings [33].

\section{Summary and Outlook}

The LHCb experiment has performed very well throughout the 2010 LHC run. About $37 \mathrm{pb}^{-1}$ of data have been recorded, with a data taking efficiency in excess of $90 \%$. A number of early 
analyses have demonstrated the ability of the $\mathrm{LHCb}$ detector to produce high quality results under harsher conditions than the experiment was designed for. Key performance parameters match or are close to expectations from simulation studies. Further improvements are still expected from better calibration and alignment. In several key analyses, sensitivities approaching those of existing measurements can already be expected from the 2010 data sample. With the much larger data sample expected for 2011, LHCb will be able to probe for New Physics signatures. Exciting times are ahead of us.

\section{References}

[1] B. Adeva et al. [LHCb Collaboration], "Roadmap for selected key measurements of LHCb", arxiv:0912.4179v2 [hep-ex].

[2] A.A. Alves Jr. et al. [LHCb Collaboration], "The LHCb Detector at the LHC”, 2008 JINST 3 S08005.

[3] F. Alessio [on behalf of the LHCb Collaboration], "LHCb Operation and Performance", these proceedings.

[4] A. Contu [on behalf of the LHCb Collaboration], "Particle Production Studies at LHCb", these proceedings.

[5] D. Hutchcroft [on behalf of the LHCb Collaboration], " $W$ and $Z$ production in the Forward Region", these proceedings.

[6] G. Auriemma [on behalf of the LHCb Collaboration], "Inclusive Jets and Dijets in LHCb", these proceedings.

[7] The LHCb Collaboration, "Prompt charm production in $p p$ collisions at $\sqrt{s}=7 \mathrm{TeV}$ ", LHCb-CONF-2010-013

[8] P. Hunt [on behalf of the LHCb Collaboration], "Prospects for charm physics", these proceedings.

[9] P. Robbe [on behalf of the LHCb Collaboration], "Prompt $J / \psi$ and $b \rightarrow J / \psi X$ production in $p p$ collisions at $\mathrm{LHCb}$ ", these proceedings.

[10] R. Aaij et al. [LHCb Collaboration], "Measurement of $J / \psi$ production in $p p$ collisions at $\sqrt{s}=7 \mathrm{TeV}$ ", submitted to Eur. Phys. J. C, arXiv:1103.0423 [hep-ex].

[11] T. Sjöstrand, S. Mrenna and P.Z. Skands, J. High Energy Phys. 0605 (2006) 026, arXiv:hep-ph/0603175.

[12] D.J. Lange, Nucl. Instrum. and Meth. A 462 (2001) 152.

[13] P. Abreu et al. [DELPHI Collaboration], Phys. Lett. B 341 (1994) 109; O. Adriani et al. [L3 Collaboration], Phys. Lett. B 317 (1993) 467; D. Buskulic et al. [ALEPH Collaboration], Phys. Lett. B 295 (1992) 396.

[14] R. Aaij et al. [LHCb Collaboration], Phys. Lett. B 694 (2010) 209

[15] M. Gronau and D. London, Phys. Lett. B 253 (1991) 483; M. Gronau and D. Wyler, Phys. Lett. B 265 (1991) 172.

[16] D. Atwood, I. Dunietz and A. Soni, Phys. Rev. Lett. 78 (1997) 3257; D. Atwood, I. Dunietz and A. Soni, Phys. Rev. D 63 (2001) 036005. 
[17] A. Giri, Y. Grossman, A. Soffer and J. Zupan, Phys. Rev. D 68 (2003) 054018; A. Bondar and A. Poluektov, Eur. Phys. J. C 47 (2006) 347; A. Bondar and A. Poluektov, Eur. Phys. J. C 55 (2008) 51.

[18] A. Martens [on behalf of the LHCb Collaboration], "Studies of Hadronic B Decays with Early LHCb Data", these proceedings.

[19] The CDF Collaboration, "An Updated Measurement of the CP Violating Phase $\beta_{s}^{J / \psi \phi}$ in $B_{s}^{0} \rightarrow J / \psi \phi$ Decays using $5.2 \mathrm{fb}^{-1}$ of Integrated Luminosity”, CDF Public Note 10206.

[20] D0 Collaboration, "Updated Measurement of the CP-Violating Phase $\phi_{s}^{J / \psi \phi}$ Using Flavor-tagged Decay $B_{s}^{0} \rightarrow J / \psi \phi ”$, D0 Note 6098-CONF.

[21] B. Khanji [on behalf of the LHCb Collaboration], "Prospects for CP Violation in $B_{s}^{0} \rightarrow J \psi \phi$ from First LHCb Data", these proceedings.

[22] M. Blanke, A. Buras, D. Guadagnoli and C. Tarantino, JHEP 0610:003 (2006).

[23] O. Buchmüller et al., Eur. Phys. J. C 64 (2009) 391; arXiv:0907.5568v1 [hep-ph].

[24] K. Nakamura et al. [Particle Data Group], J. Phys. G 37 (2010) 075021.

[25] V. Abazov et al. [D0 Collaboration], Phys. Lett. B 693 (2010) 539; arXiv:1006.3469v2 [hep-ex].

[26] CDF preliminary, "Search for $B_{s}^{0} \rightarrow \mu^{+} \mu^{-}$and $B_{d}^{0} \rightarrow \mu^{+} \mu^{-}$Decays in $3.7 \mathrm{fb}^{-1}$ of $p \bar{p}$ Collisions with CDFII", CDF Public Note 9892.

[27] R. Fleischer, N. Serra and N. Tuning, Phys. Rev. D 82 (2010) 034038.

[28] R. Aaij et al. [LHCb Collaboration], "Search for the rare decays $B_{s}^{0} \rightarrow \mu^{+} \mu$ and $B^{0} \rightarrow \mu^{+} \mu$, submitted to Phys. Lett. B, arxiv:1103.2465v1 [hep-ex].

[29] J. Altmannshofer et al., JHEP 0901 (2009) 019; F. Kruger and J. Matias, Phys. Rev. D71 (2005) 094009; U. Egede et al., JHEP 032 (2008) 0811.

[30] B. Aubert et al., Phys. Rev. D 79 (2009) 031102(R), arXiv:0804.4412 [hep-ex].

[31] J.T. Wei et al., Phys. Rev. Lett. 103 (2009) 171801, arXiv:0904.0770v2 [hep-ex].

[32] T. Aaltonen et al., Phys. Rev. D 79 (2009) 011104(R), arXiv:0804.3908v1 [hep-ex].

[33] A. Pellegrino [on behalf of the LHCb Collaboration], "Search for New Physics in Heavy Flavour Rare Decays", these proceedings. 